\title{
A Domain Ontology for Mechanical Circulatory Support Systems
}

\author{
C De Lazzari ${ }^{*}$, E Guerrieri $^{+}$, DM Pisanelli ${ }^{+}$ \\ ${ }^{*}$ CNR - Istituto di Tecnologie Biomediche, Rome, Italy \\ ${ }^{+} \mathrm{CNR}$ - Istituto di Scienze e Tecnologie della Cognizione, Rome, Italy
}

\begin{abstract}
Heart failure is a major health problem with a high mortality rate in industrialized countries. Its ultimate therapy, heart transplantation, is limited by the shortage of heart donors. Since decades researchers have been working to solve this problem by developing Mechanical Circulatory Support Systems (MCSS) that can replace or assist the failing heart. We defined an ontology - i.e. a catalogue of existing items with formal specification of their features -in the domain of MCSS. Our aim is to support the implementation of an advanced database performing an intelligent management of MCSS installed in a given department. Our database, and relative ontology, has been realized by means of the Protégé2000 tool. About 30 different types of MCSS have been classified and represented according to their qualities. The tool is able to perform several kinds of queries, both with respect to the kind of machine requested and with respect to actual machines installed in a given ward or department.
\end{abstract}

\section{Introduction}

Heart failure is a condition that affects more than 20 million people around the world [1], especially in industrialized countries (nearly 5 million in the United States [2]). The failure usually happens when the heart becomes damaged and weak. Eventually, the heart is unable to pump enough blood to meet the body's needs.

Cardiac pathologies rank number one as cause of hospitalization for people aged 65 and more in USA [3], whereas in Italy cardiovascular diseases account for $43 \%$ of the totality of deaths [4]. Among this class of diseases, heart failure is actually the most relevant, since almost every cardiac disorder may lead - and usually leads - to heart failure.

The ultimate therapy for such a failure is heart transplantation, but it is limited by the shortage of heart donors. Since decades researchers have been working to solve this problem by developing Mechanical Circulatory Support Systems (MCSS) that can replace or assist the failing heart.

Nowadays there is a great variety of MCSS available in the market and this is certainly a positive thing, but it may also create confusion among the decision makers who have the choose the optimal solution for their needs. The major problem concerning such a difficult choice is the lack of uniformity in the information available in this field of cardiovascular medicine.

This is the main reason why we defined an ontology in the domain of MCSS. An ontology is a catalogue of existing items with formal specification of their features. Our aim is to support the implementation of an advanced database performing an intelligent management of MCSS installed in a given department.

\section{Materials and methods}

Apart from its definition in a philosophical context where it refers to the subject of existence - ontology in information science is "a partial specification of a conceptualization", as stated by Guarino [5], whereas Sowa proposed the following definition influenced by Leibniz [6]:

\begin{abstract}
"The subject of ontology is the study of the categories of things that exist or may exist in some domain. The product of such a study, called an ontology, is a catalogue of the types of things that are assumed to exist in a domain of interest $\mathrm{D}$ from the perspective of a person who uses a language $\mathrm{L}$ for the purpose of talking about D."
\end{abstract}

Actually there is some disagreement on what is an ontology. Some admit informal descriptions and hierarchies, only aimed at organizing some uses of natural language; others require that an ontology be a theory, i.e. a formal vocabulary with axioms defined on such vocabulary, possibly with the help of some axiom schema, as in description logics.

In our perspective, an ontology is a formal model which specifies the conceptualization (i.e. the intended meaning) of a lexical item as it is used in a certain domain.

Our ontology of MCSS is therefore a model of the features of such systems. In such a model the different machines (the "lexical items", i.e. the way people in 
biomedical domain call them) are represented together with their main characteristics.

In order to implement our ontology, we employed the Protégé-2000 tool, developed at Stanford University [7]. It is a powerful open-source Java-based tool that allows the user to build a domain ontology, to customize data entry forms and enter data.

We took profit also from Protégé's advanced graphical features, which enabled us to develop a user-friendly graphical environment for data input and queries.

\section{Results}

About 30 different types of MCSS (e.g. Abiomed BVS 5000, HeartMate 1000, Novacor N100) have been classified in the system (Fig. 1).

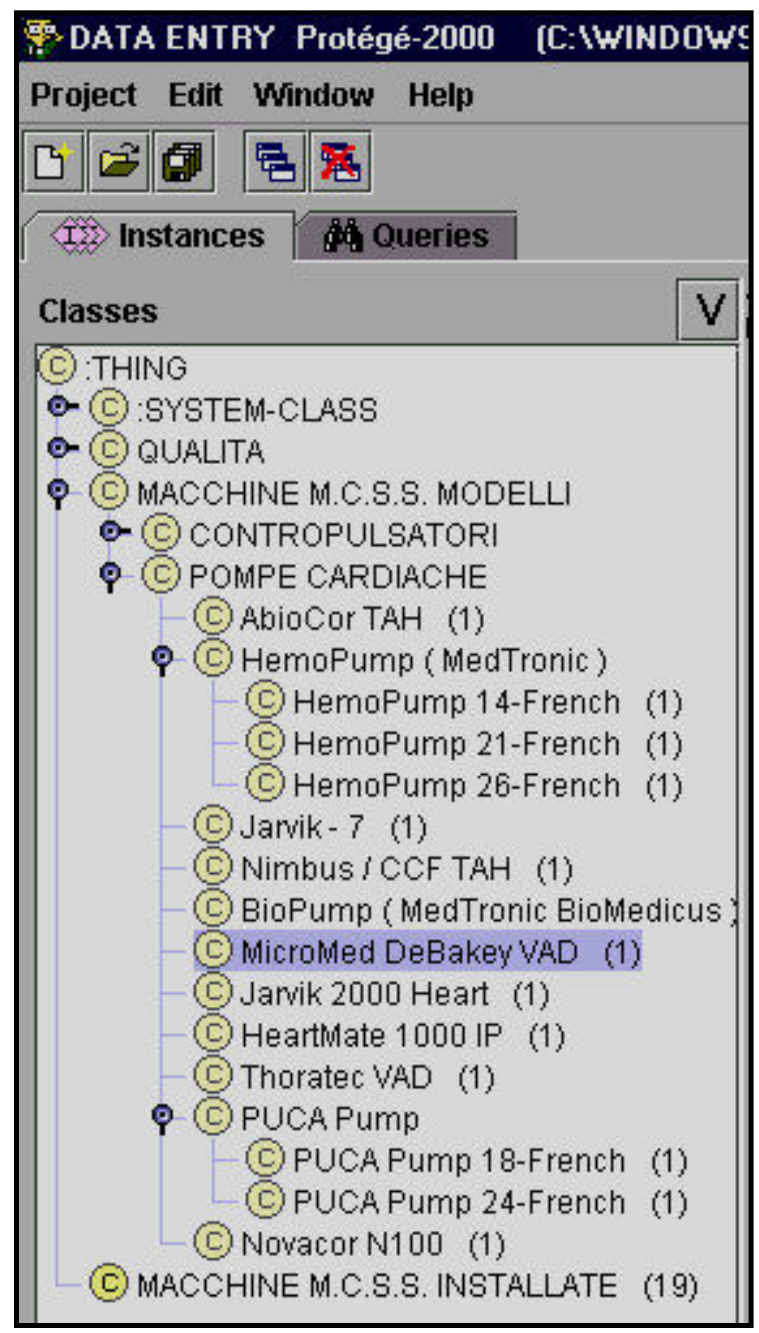

Figure 1. Fragment of the Protégé-2000 window reporting the MCSS models classified in the system.
They have been represented (Fig. 2) according to their features (e.g. pulsatile or continuous flow, synchronized or not with ECG, right, left or bi-ventricular assistance).

\begin{tabular}{|c|c|c|}
\hline \multicolumn{3}{|c|}{ I) MicroMed DeBakey VAD [MicroMed DeBakey Vad] } \\
\hline Nome Del Macchinario & & Terapie Di Supporto \\
\hline \multicolumn{2}{|l|}{ MicroHed DeBakey VAD } & \\
\hline \multicolumn{2}{|c|}{ Principio Di Funzionamento } & \\
\hline \multicolumn{2}{|l|}{ (c) ELET TRICO } & \\
\hline Tipo Di Flusso & Flusso $1 / \min$ & \\
\hline CFLSSO CONTINUO & 6.0 & \\
\hline \multicolumn{2}{|l|}{ Tipo Di Alimentazione } & Tipo Di Assistenza \\
\hline \multicolumn{2}{|l|}{ (C) TUBI E CAVI } & GRVAD \\
\hline \multicolumn{2}{|l|}{ Tecnologia } & \\
\hline \multicolumn{2}{|l|}{ (C) AXIAL PUMP } & \\
\hline \multicolumn{2}{|c|}{ Tipo Di Installazione Pompa } & \\
\hline \multicolumn{2}{|c|}{ (c) INSTALLAZIONE INTERNA } & \\
\hline \multicolumn{2}{|l|}{ Periodo Terapeutico } & Tipo Di Sincronia \\
\hline \multicolumn{2}{|l|}{ C) BREVE TERHINE } & Non Sincronizzato \\
\hline
\end{tabular}

Figure 2. An example of the features represented for each MCSS model.

Such features are hierarchically represented in the system (Fig.3).

In our system we distinguish two main classes of MCSS: "MCSS models" and "MCSS installed". The former accounts for the features of the different models, independently from the actual machines. The latter represents those machines actually installed in a given department.

In such a way, the tool is able to perform basically two types of queries, i.e. with respect to the kind of machine requested (given a set of qualities, retrieve those systems satisfying those features, Fig.4) and with respect to actual machines installed in a given ward or department.

Such an asset is a peculiar result of the ontological approach, being the tool is able to perform knowledge management at an "abstract" level (retrieving classes of machines according to desired features) and at a "concrete" level (retrieving actual machines according to desired features and availability). 


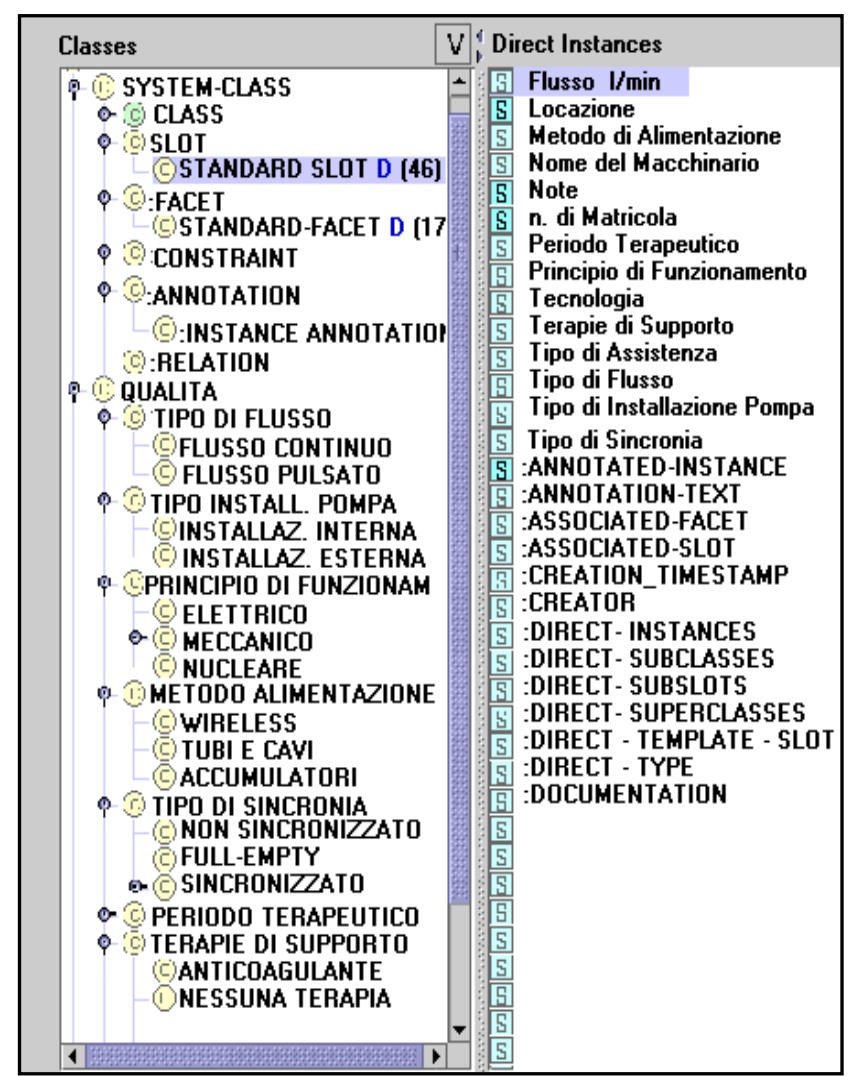

Figure 3. Hierarchy of MCSS features ("qualità" in Italian).

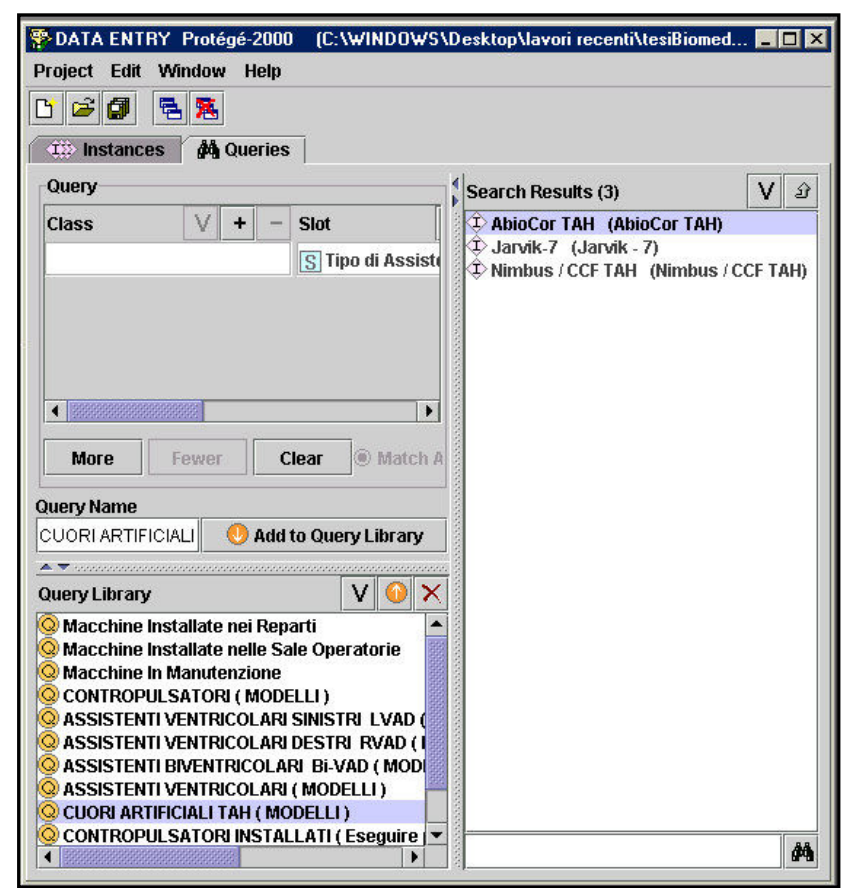

Figure 4. An example of query for retrieving the models of Total Artificial Heart (TAH) reported in our system.
The very same system can be used both as a database of MCSS installed in a given health-care structure and as a knowledge base representing performances and characteristics of the different MCSS models.

\section{Conclusions}

We took profit from the advanced features of the Protégé-2000 tool, which enabled us to develop a userfriendly graphical environment for data, input and queries. But the most relevant asset of our system is the integration of a database and a domain ontology.

Although it is recognized that ontologies may help building better and more interoperable information systems, there is skepticism on the real impact they may have in the future. We believe that ontologies will succeed in the information system arena and no systems will ever be designed without an ontological approach.

\section{References}

[1] Congestive heart failure worldwide markets, clinical status and product development opportunities. New Medicine, Inc. 1997; 1-40.

[2] American Heart Association. Heart and Stroke Statistical Update. 2001

[3] Ho KKl, Pinski JL, Kannel WB, et al. The epidemiology of heart failure: the Framingham Study. J Am Coll Cardiol. 1993;22(supp A):6A-13A

[4] Annuario Statistico Italiano (ISTAT) 2002.

[5] Guarino N Formal Ontology and Information Systems, in Guarino N (ed.). Formal Ontology in Information Systems, Amsterdam, IOS-Press, 1998.

[6] Sowa J, Communication to the ontology-std mailing list, 1997.

[7] http://protege.stanford.edu

Address for correspondence:

Claudio De Lazzari

CNR ITBM, Viale Marx, 15

00137 Rome, Italy.

dela@itbm.rm.cnr.it 